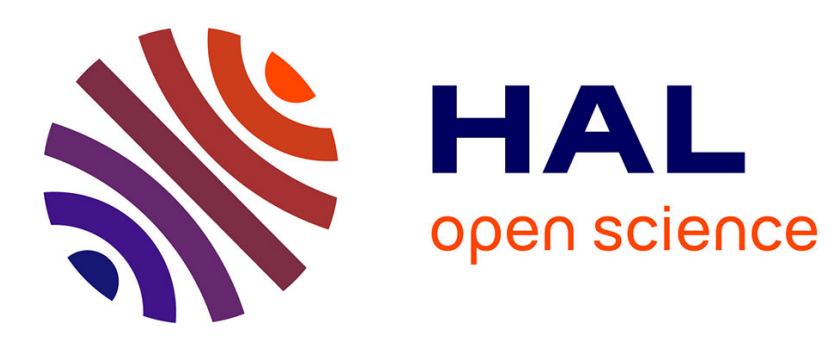

\title{
A Mixed-integer Nonlinear Optimization Approach for Well Placement and Geometry
}

C Lizon, C d'Ambrosio, Leo Liberti, Mickaële Le Ravalec, Delphine Sinoquet

\section{To cite this version:}

C Lizon, C d'Ambrosio, Leo Liberti, Mickaële Le Ravalec, Delphine Sinoquet. A Mixed-integer Nonlinear Optimization Approach for Well Placement and Geometry. ECMOR XIV -14 th European Conference on the Mathematics of Oil Recovery, Sep 2014, Catania, Italy. hal-02284385

\section{HAL Id: hal-02284385 \\ https://hal-ifp.archives-ouvertes.fr/hal-02284385}

Submitted on 11 Sep 2019

HAL is a multi-disciplinary open access archive for the deposit and dissemination of scientific research documents, whether they are published or not. The documents may come from teaching and research institutions in France or abroad, or from public or private research centers.
L'archive ouverte pluridisciplinaire HAL, est destinée au dépôt et à la diffusion de documents scientifiques de niveau recherche, publiés ou non, émanant des établissements d'enseignement et de recherche français ou étrangers, des laboratoires publics ou privés. 


\title{
Th A38
}

\section{A Mixed-integer Nonlinear Optimization Approach for Well Placement and Geometry}

\author{
C. Lizon* (IFPEN), C. D'Ambrosio (CNRS and LIX Ecole Polytechnique), L. \\ Liberti (IBM T.J. Watson and LIX Ecole Polytechnique), M. Le Ravalec \\ (IFPEN) \& D. Sinoquet (IFPEN)
}

\section{SUMMARY}

Optimizing well configuration within oil fields usually consists in maximizing profits from oil production in a long-term horizon. Such profits are typically predicted using CPU-time demanding fluid flow simulations. The variables of the optimization problem considered in this work are the number of wells, their locations and types, as well as the number and trajectories of the branches drilled from the given producers.

We propose a methodology that reduces the complexity and the underlying simulation cost of this optimization problem. First, we introduce various physical constraints related to the distance between wells and oil bearing areas in order to select a suitable region for drilling.

A direct search method is then coupled with surrogate models approximating the objective function to obtain a good estimation of the optimal number, location and type of wells while limiting the simulations' number.

Given the solution determined in the previous step, we analyze the responses provided by the fluid flow simulator (e.g., oil saturation distribution) and apply a mixed-combinatorial method to define the geometry of the rectilinear well branches. This makes it possible to improve the current solution. The potential of the second step of the proposed methodology is evaluated with a synthetic twodimensional reservoir model. 


\section{Introduction}

As oil reserves are declining, a strong effort is required in petroleum industry to propose efficient extraction techniques able to increase field production. In this context, developing efficient simulation-based optimization methods for well placement and monitoring is a key challenge. Thanks to intelligent fields or Smart fields (van den Berg et al. (2010); AbdulKarim et al. (2010)), a large amount of information can be used to improve our knowledge of the reservoir, in particular to improve our numerical models for generating oil production forecasts. These models, calibrated with history matching (Schulze-Riegert and Ghedan (2007); Oliver et al. (2008); Le Ravalec et al. (2012)), have better forecasting capabilities and can be used to make decisions related to the development of oil fields and thus have a more efficient field exploitation.

One of these decisions is the location and geometry (i.e. trajectory) of wells. Optimizing the number of wells, their trajectory, and their type (injector or producer) is a difficult problem and can be very costly in terms of fluid flow reservoir simulations. Optimizing well location has been observed to yield non-smooth cost functions (Bouzarkouna et al. (2010); Isebor et al. (2013)). Hence, in optimal well placement one should perform some kind of global search that prevents the optimization from being trapped in solutions that are not acceptable in practice (e.g. "low" Net Present Value (NPV) or total oil production).

In the first section we propose a two-step methodology to solve this challenging problem. The first step consists in solving a Black-Box Mixed Integer Non-Linear Problem (MINLP) with a Pattern Search method (Audet and Dennis (2001); Le Digabel (2011)). This method belongs to the class of heuristic optimization methods that were adapted for Black-Box MINLP problems, but may require a large number of evaluations. The variables of this first optimization problem are the number of wells, their locations and types. We optimize the configuration of vertical wells, this simplification allowing to reduce the number of variables. In order to limit the number of evaluations, the Pattern Search method is coupled with metamodels based upon a Gaussian process approach (Gramacy and Le Digabel (2011)). Simulation outputs of the obtained solution are used in the second step of the methodology.

In this second step, starting from the optimized vertical well configuration, we solve a simplified problem to optimize trajectories of vertical wells by adding branches. Only two simulations are required in this phase, one to collect reference data from the initial well configuration (optimized result of the first step), and the second one to validate the solution obtained in this second step. The simplifications we propose lead to a convex MINLP problem that can be solved by an exact method as Branch-and-Bound (Dakin (1965); Gupta and Ravindran (1985)).

The two phases of the methodology are described in the first section. The second section focuses on the mathematical model built for the simplified MINLP problem of well branching. Finally, we present preliminary computational results of the second step of the methodology obtained for a two-dimensional case.

\section{Methodology}

In this section, we describe the proposed methodology: first, the Black-Box MINLP optimization problem for vertical well placement, then, the analysis of the obtained solution and the definition of the input of the second optimization problem for well branching, and lastly, the objectives of this second optimization problem.

\section{Optimization of vertical wells: type and location}

In the first optimization, we limit the number of optimized variables by considering a limited number of wells and only vertical trajectories. The optimized objective function is the Net Present Value (NPV) 
defined as follows

$$
\begin{aligned}
f_{N P V} & =\sum_{n=1}^{N_{I}+N_{P}}\left(C^{F} l_{n} \log \left(l_{n}\right)+C^{D}\right) \\
& +\sum_{t=1}^{T} \frac{1}{(1+\mathrm{R})^{t}}\left(\sum_{n=1}^{N_{P}}\left(C^{P O} q_{t n}^{P O}-C^{P W} q_{t n}^{P W}\right)-\sum_{n=N_{P}+1}^{N_{P}+N_{I}}\left(C^{I W} q_{t n}^{I W}\right)\right),
\end{aligned}
$$

where

- $N_{I}$ and $N_{P}$ are the number of injectors and producers,

- $C^{D}$ is the drilling cost and $C^{F} l_{n} \log \left(l_{n}\right)$ accounts for the extra cost related to the length of well $n$ (namely $l_{n}$ ),

- $q_{t n}^{P O}, q_{t n}^{P W}$ and $q_{t n}^{I W}$ are, respectively, produced oil, produced water (for producer well $n$ ) and injected water (for injector well $n$ ) cumulated quantities during production period $t$, computed by a fluid flow reservoir simulator,

- $R$ is a discount factor (it implies that early oil production is assigned a stronger weight in the NPV function than the late production) and $T$ is the number of production periods,

- $C^{P O}, C^{P W}$ and $C^{I W}$ are positive weights associated with each term.

Maximizing this objective function with respect to the number of active wells (producers and injectors), their location and their length is a Black-Box MINLP optimization problem coupled with a computationally expensive simulator, namely the fluid flow reservoir simulator. Since the NPV function is generally non-smooth and non-convex and gradient information is rarely available, its minimization requires derivative-free global optimization methods (Bouzarkouna et al. (2010); Echeverría Ciaurri et al. (2011); Isebor et al. (2013); Onwunalu and Durlofsky (2010)). As these methods require a large number of simulations, we propose to couple the optimization method with meta-modeling techniques to replace costly simulations by an approximated response surface. Gramacy and Le Digabel (2011) proposed a direct search method coupled with Gaussian process surrogates that permits the introduction of dedicated strategies in the exploration phase, based on surrogate predictions. Gaussian processes provide not only a surrogate model of the objective function to be minimized/maximized, but also an estimate of the predictive variance. This can be useful for selecting the most promising candidates to be simulated in the poll step of the mesh adaptive direct search.

This step provides a first optimized configuration with production and injection vertical wells. The outputs of the fluid flow reservoir simulation obtained with this configuration are analyzed to determine the areas that were not produced.

\section{Analysis of optimized vertical well configuration}

In practice, reservoir engineers make the decision to add wells or branches from the analysis of the outputs of the fluid flow simulator. The outputs that are commonly considered are physical attributes, such as oil saturation or oil thickness. We combine this pragmatic approach with optimization to better determine the trajectory of wells with branches. We analyze the outputs of the simulator and attribute a score to distinguish regions of the field: a high score means that an area has good drilling potential. For simplicity, we focused on a single attribute called $H_{u} \phi S_{o}$ which is the product of the utility thickness $\left(H_{u}\right)$, the porosity $(\phi)$, and the oil saturation $\left(S_{o}\right) . H_{u} \phi S_{o}$ quantifies the volume of oil still not produced. An example of a two-dimensional map, obtained with $H_{u} \phi S_{o}$ is displayed in Figure 1. This map is obtained from the outputs of a fluid flow reservoir simulation after producing the field for 4 years with one vertical well at the center of the domain. In this map, we observe that there is still oil not produced. 
We also see that some cells have a high $H_{u} \phi S_{o}$ but are spatially close to cells with low $H_{u} \phi S_{o}$ values. Clearly, such cells are not interesting targets compared to zones corresponding to a group of cells with more homogeneous and relatively high $H_{u} \phi S_{o}$ values. In order to reduce the number of variables, and to avoid isolated valued, scores are computed for groups of cells. Such groups of cells are called areas in the remaining of this paper.

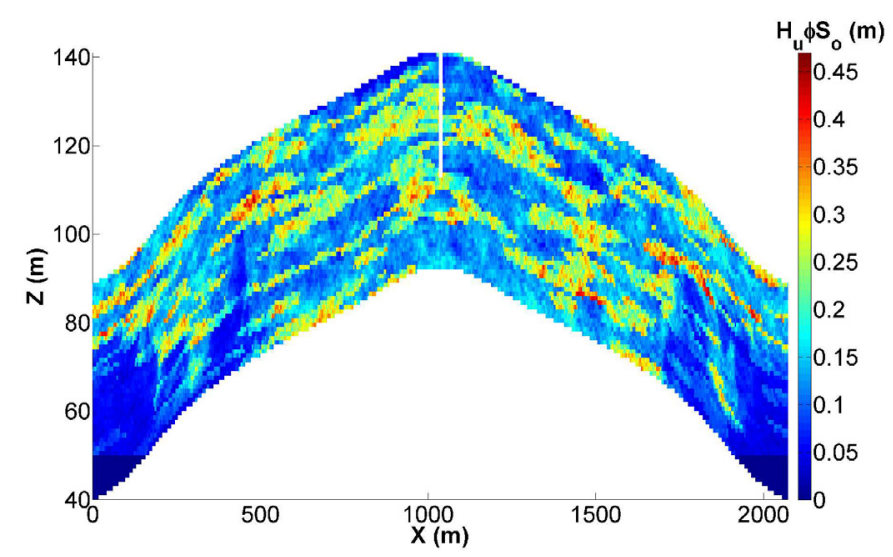

Figure $1 H_{u} \phi S_{0}$ map associated with a single vertical well configuration considered later in the optimization case

Another property that could be taken into account in the definition of high potential areas is the connectivity between cells. Based on connectivity consideration, we can introduce here of forbidden zones, i.e, parts of the reservoir we cannot physically drill or that we do not want to drill because, in general, they are not well connected.

From this analysis, we determine the input data to be used within the following step of the proposed methodology, which is the branching optimization problem.

\section{Optimization of well trajectory: branching problem}

Starting from a vertical well configuration, given as the solution of the first optimization problem described above, we want to improve oil production by adding branches to the existing vertical wells. A branch sub-problem is introduced: given a well configuration without branches, we aim at finding the potential branches that can be drilled from each well. We focus on branches from production wells only. The vertical injection wells obtained in the first optimization solution are fixed and kept unchanged in this optimization.

In order to obtain a very efficient methodology, in this optimization we do not use reservoir fluid flow simulation. For this reason we consider a simplified objective function compared to the NPV function of the first optimization problem. This simplified objective function is an estimation of the oil production based on the $H_{u} \phi S_{o}$ values of the cells belonging to all areas connected to the production wells by a branch. An additional constraint is introduced to account for the branch construction cost, which in turn depends on the branch length, to avoid well configuration with an unacceptable drilling cost.

As described in the next section, for modeling purposes we introduce the concept of cluster. A cluster is a set of oil areas that are "close enough" to be reached by a single branch (close enough has to be understood in terms of connectivity). The mathematical model below can be used to aggregate areas into clusters and to determine how to reach a cluster with a branch from a well, with an acceptable construction cost. We define the end of a branch as a cluster reference point, i.e. a point that is close enough to each area of the cluster (the branch thus obtained can be expected to produce a significant amount of the oil in the cluster). 


\section{Mathematical model of well branching problem}

This section describes the mathematical model designed to solve the branching problem. It is organized as follows: we first present the notations, namely the sets (represented by Greek letters), the input data or parameters (upper capitals), the variables (lower capitals), and the simple bounds on variables. We conclude the section by discussing the objective function and the set of constraints, that are properly reformulated to obtain a convex MINLP problem that can be solved by exact optimization methods as discussed in last section. For the sake of simplicity, we restrict the model to a two-dimensional case (however this formulation can be readily generalized to three-dimensional problems).

\section{Input Data}

Sets:

- $\Phi$ : set of wells in the initial solution.

- П: set of areas of the reservoir representing the cells with a high score at the end of the simulation with the initial well configuration.

- $K$ : maximum number of clusters.

- $\Gamma=\{1, \ldots, K\}$ : set of clusters.

Parameters:

- $X_{i d}$ : coordinates of the center of oil area $i(i \in \Pi, d \in\{1,2\})$.

- $O_{i}$ : volume of oil in area $i(i \in \Pi)$, computed from $H_{u} \phi S_{o}$ values in the corresponding area.

- $\left[\underline{X}_{d}, \bar{X}_{d}\right]$ : oil reservoir domain for dimension $d(d \in\{1,2\})$.

- $\underline{L}, \bar{L}$ and $H$ : minimum and maximum lengths of a branch, and maximum length of all branches built. The third parameter is necessary to limit the construction cost based on the target budget.

- $R$ : maximum distance between oil area and branch end, i.e., maximum distance of a point in $\Pi$ with respect to the cluster reference point.

- $V_{n d}$ : coordinates of the top of well $n$ for dimension $d(d \in\{1,2\}, n \in \Phi)$.

- $W_{n}$ : height of well $n(n \in \Phi)$, we assume that there are vertical wells only.

- $B$ : maximum number of branches per well.

\section{Variables}

Continuous:

- $z_{j d}$ : $d$-th coordinate of the reference point for cluster $j(j \in \Gamma, d \in\{1,2\})$.

- $w_{j n}$ : height of the junction point on well $n$ connecting cluster $j$ through a branch $(j \in \Gamma, n \in \Phi)$.

- $s_{j n}$ : square of the distance between the reference point of cluster $j$ and the junction point of well $n(j \in \Gamma, n \in \Phi)$. 
Binary:

- $q_{j n}$ : activation of connection between cluster $j$ and well $n(j \in \Gamma, n \in \Phi)$ : [1 if active, 0 otherwise].

- $x_{i j}$ : inclusion of oil area $i$ in cluster $j(i \in \Pi, j \in \Gamma)$ : [1 if included, 0 otherwise].

- $y_{j}$ : activation of cluster $j(j \in \Gamma)$, an inactive cluster is not linked to any well: [1 if active, 0 otherwise].

Some additional parameters and variables will be introduced later to formulate the optimization as a convex MINLP problem.

\section{Simple Bounds}

Simple bound on continuous variables are defined to ensure that the well configurations obtained are specified within the physical domain considered.

$$
\begin{aligned}
0 \leq w_{j n} \leq W_{n} & \forall j \in \Gamma, n \in \Phi, \\
\underline{Z}_{d} \leq z_{j d} \leq \bar{Z}_{d} & \forall j \in \Gamma, d \in\{1,2\}, \\
0 \leq s_{j n} \leq\left\|\bar{X}_{d}-\underline{X}_{d}\right\|_{2}^{2} & \forall j \in \Gamma, n \in \Phi .
\end{aligned}
$$

\section{Constraints}

$$
\begin{array}{rl}
\sum_{j \in \Gamma, n \in \Phi} q_{j n} s_{j n} \leq H^{2}, & \\
q_{j n} \underline{L}^{2} \leq q_{j n} s_{j n} & \forall j \in \Gamma, \forall n \in \Phi, \\
q_{j n} s_{j n} \leq q_{j n} \bar{L}^{2} & \forall j \in \Gamma, \forall n \in \Phi, \\
\sum_{j \in \Gamma} x_{i j} \leq 1 & \forall i \in \Pi, \\
x_{i} \leq y_{j} & \forall i \in \Pi, j \in \Gamma, \\
y_{j} \leq \sum_{i \in \Pi} x_{i j} & j \in \Gamma, \\
q_{j n} \leq y_{j} & \forall n \in \Phi, j \in \Gamma, \\
\sum_{j \in \Gamma} q_{j n} \leq B & \forall n \in \Phi, \\
\sum_{n \in \Phi} q_{j n} \leq y_{j} & \forall j \in \Gamma, \\
\sum_{d \in\{1,2\}}\left(X_{i d}-z_{j d}\right)^{2} \leq R^{2}+M\left(1-x_{i j}\right) & \forall j \in \Gamma, i \in \Pi, \\
z_{j 2} \leq V_{n 2}+w_{j n}+\left(1-q_{j n}\right) \bar{Z}_{2} & \forall j \in \Gamma, n \in \Phi, \\
s_{j n} \geq\left(V_{n 1}-z_{j 1}\right)^{2}+\left(\left(V_{n 2}+w_{j n}\right)-z_{j 2}\right)^{2} & \forall j \in \Gamma, n \in \Phi, \\
z_{j 1} \leq z_{k 1} & \forall j<k \in \Gamma .
\end{array}
$$

Equation (5) represents a budget constraint. As anticipated at the beginning of this section, this constraint is introduced to limit the cost associated with the branches. The bilinear terms $q_{j n} s_{j n}$ will be reformulated at the end of the section to obtain a linear constraint plus auxiliary variables and linear constraints.

Equations (6) and (7) represent bound inequalities for the branch length. 
By means of equations (8) each area $i$ is assigned at most to one cluster. Equations (9) ensure that area $i$ is assigned to cluster $j$ only if this cluster is active. Equations (10) imply that cluster $j$ is not active if no area is assigned to it.

Equations (11) allow cluster $j$ to be connected to well $n$ only if this cluster is active, while equations (12) limit the number of branches for each well to $B$. Additionally, constraint (13) indicates that cluster $j$ can be connected to at most one well, only if the cluster is active.

As mentioned earlier, the oil-bearing areas have to be close enough to the reference point of a cluster to be assigned to it. In such a case, we can expect that a significant amount of the oil contained in these areas is produced through the branch corresponding to the cluster. Equations (14) ensure that, if area $i$ is assigned to cluster $j$, the distance between the cluster reference point and the area cannot be larger than $R$.

Equations (15) represent an angle constraint between the well mainbore and a branch (if cluster $j$ is connected to well $n$ ). Note that since all wells are vertical, forcing the height of the well junction point to be greater than the height of the cluster reference point is the same as setting a $90^{\circ}$ upper bound on the branch angle. Equation (16) is related to the square distance between cluster reference point $j$ and its junction point on well $n$. Equations (17) establish symmetry breaking constraints, i.e., they impose an order for the lateral coordinates of the clusters ${ }^{1}$.

\section{Objective function}

The model that is proposed here aims at maximizing the total amount of oil produced. Formally, this quantity can be estimated through the linear objective function:

$$
\max \sum_{i \in \Pi, j \in \Gamma, n \in \Phi} x_{i j} q_{j n} O_{i}
$$

which accounts for the oil associated with areas belonging to a connected cluster.

\section{Reformulations}

We can simplify a number of constraints with the help of auxiliary variables: $r_{j n}$ and $u_{j n i}$ replace the bilinear terms $q_{j n} s_{j n}(j \in \Gamma, n \in \Phi)$ and $x_{i j} q_{j n},(j \in \Gamma, n \in \Phi, i \in \Pi)$ respectively. The variable $r_{j n}$ is the square of the distance between a connected cluster $j$ and well $n$ (if cluster $j$ is not connected, $r_{j n}=0$ ). The binary variable $u_{j n i}$ indicates that area $i$ belongs to cluster $j$ which is connected to well $n$ (if oil area $i$ is not produced, $u_{j n i}=0$ ).

The relation between the original and the new variables is given by the following linear constraints:

$$
\begin{aligned}
r_{j n} \leq s_{j n}+M\left(1-q_{j n}\right) & \forall j \in \Gamma, n \in \Phi, \\
r_{j n} \geq s_{j n}-M\left(1-q_{j n}\right) & \forall j \in \Gamma, n \in \Phi, \\
r_{j n} \geq q_{j n} \bar{L}^{2} & \forall j \in \Gamma, n \in \Phi, \\
r_{j n} \leq q_{j n} \underline{L}^{2} & \forall j \in \Gamma, n \in \Phi, \\
u_{j n i} \leq q_{j n} & \forall n \in \Phi, j \in \Gamma, i \in \Pi, \\
u_{j n i} \leq x_{i j} & \forall n \in \Phi, j \in \Gamma, i \in \Pi, \\
u_{j n i} \geq q_{j n}+x_{i j}-1 & \forall n \in \Phi, j \in \Gamma, i \in \Pi .
\end{aligned}
$$

where $M$ is defined as the square of the maximum distance between two points in the reservoir.

\footnotetext{
${ }^{1}$ Note that these symmetry breaking constraints do not remove any feasible solution (they keep only one configuration when several are equivalent). This limits the feasible space of the solution to be explored by the optimization algorithm considered.
} 
According to equations (18), (19), (20) and (21), if cluster $j$ is connected to well $n$ (i.e. $q_{j n}=1$ ), then the corresponding branch length cannot be larger than $\bar{L}$ and smaller than $\underline{L}$. If cluster $j$ is not connected to well $n$ (i.e. $q_{j n}=0$ ), equations (20) and (21) imply $r_{j n}=0$, and (18) and (19) are satisfied.

In terms of the new variables, the budget constraint (5) can be rewritten as the linear constraint:

$$
\sum_{j \in \Gamma, n \in \Phi} r_{j n} \leq H^{2}
$$

In addition, the objective function is now linear:

$$
\max \sum_{i \in \Pi, j \in \Gamma, n \in \Phi} u_{j n i} O_{i}
$$

Constraints (6) and (7) are also rewritten as linear constraints, which are constraints (20) and (21).

As a consequence, the optimization problem formulated in terms of the new variables is a convex MINLP.

It is important to note that in the formulation above we impose no restriction on the crossing or proximity of branches. These constraints are very important in practice but would complicate the problem considerably, and transform it into a non convex MINLP, which is much harder to solve. These constraints could be dealt by means of validation and post-processing stages: identifying violation of crossing constraints and recovering feasibility is easy in two-dimensional case.

\section{Computational results}

This section shows preliminary results that illustrate the second phase of our methodology. We investigate the potential of the proposed method using a relatively simple reservoir model based on a twodimensional discretization. The reservoir corresponds to an anticline trap with an aquifer at the bottom to ensure pressure support. The reservoir model has of $830 \times 1 \times 50$ grid blocks, and the dimensions of each grid block are $D X=2.5 \mathrm{~m}, D Y=10 \mathrm{~m}$, and $D Z=1 \mathrm{~m}$. The reservoir presents two facies with volume fractions $40 \%$ and $60 \%$. The mean and variance of the corresponding porosities are 0.4 and 0.005 for the first facies and 0.2 and 0.001 for the second one. A facies realization is first generated using a Fast Fourier Transform Moving Average (FFTMA) algorithm (Le Ravalec et al. (2000)) and the Truncated Gaussian method (Chiles (1984); Matheron et al. (1987)) with a Gaussian variogram characterized by a horizontal range of $1250 \mathrm{~m}$ and a vertical range of $5 \mathrm{~m}$. Thereafter, the two resulting facies are populated with porosity values applying again the FFTMA algorithm. The porosity realizations for each facies are characterized by an exponential variogram. The porosity model is converted into a permeability model using deterministic relationships between the logarithm of permeability $K$ and porosity $\phi$. For the first facies, the relation is $\log (K)=5 \phi+0.4$, and for the second facies, the relation is $\log (K)=7 \phi+0.3$. The resulting porosity and permeability models are shown in Figure 2 and Figure 3.

The fluid flow simulator used is PumaFlow ${ }^{T M}$ (PumaFlow (2012)). The initial configuration considered here is not the solution of a previous optimization. It consists of a single vertical producer located at the top of the anticline. Figure 1 represents $H_{u} \phi S_{o}$ after a production time-frame of 4 years. The cumulated oil produced is 45.39 thousand cubic meters (285 Mbbl).

In order to limit the number of variables in the model, we selected the high production potential areas, by using an approximation of $H_{u} \phi S_{o}$ based on spline interpolation, for areas of $2 \times 20$, cells and we kept areas with an $H_{u} \phi S_{o}$ score above 2.75. These areas are represented in Figure 4.

The mathematical model presented in the previous section is written in AMPL, A Mathematical Programming Language, (Fourer et al. (2003)), an algebraic modeling language widely used in the MINLP 


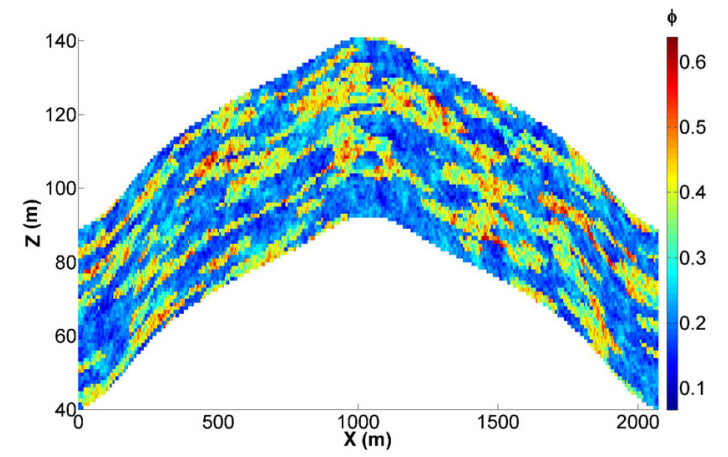

Figure 2 Porosity map

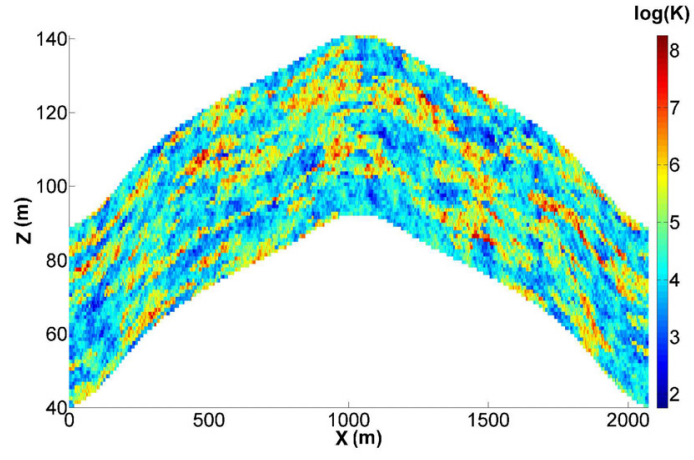

Figure 3 Permeability map (log)

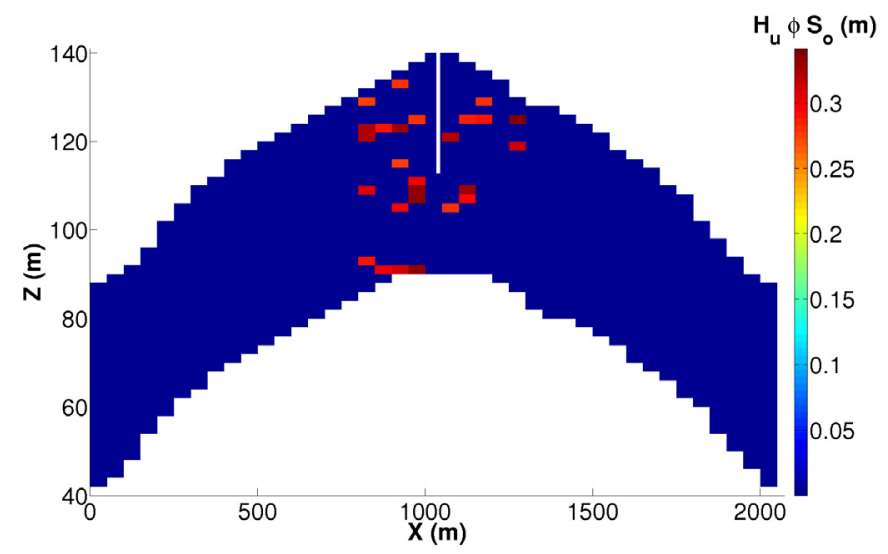

Figure 4 Map of high production potential areas.

community. The input parameters of the model are the maximum number of cluster, $K=5$, the maximum length of any branch, $\bar{L}=250 \mathrm{~m}$, and the maximum number of branches, $B=5$. The optimization problem is solved with Basic Open-Source NonLinear Mixed Integer Programming (BONMIN), a MINLP solver (Bonami et al. (2008)), using Branch-and-Bound method.

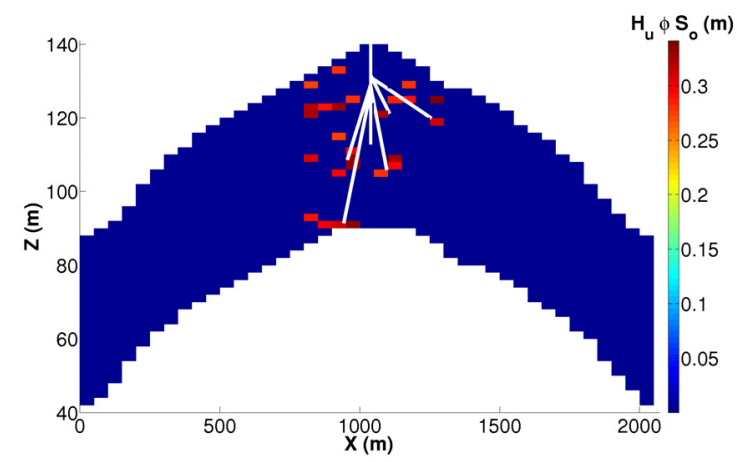

Figure 5 Optimized well trajectory with high production potential areas.

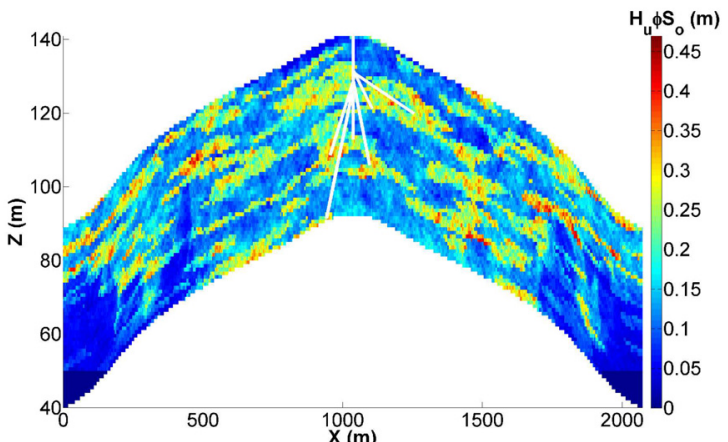

Figure 6 Optimized well trajectory with initial $H_{u} \phi S_{o}$ map.

In Figure 5 we show the optimized well trajectory where 5 branches were added. In Figure 6 this solution is superposed to the $H_{u} \phi S_{o}$ values associated with the initial vertical well configuration. The oil saturation after 4 years of production for the initial vertical well and for the optimized configuration with 5 branches are represented in Figure 7 and 8. The improvement oil recovery is apparent.

The cumulative oil produced with the new configuration with 5 branches is equal to 51.59 thousand 


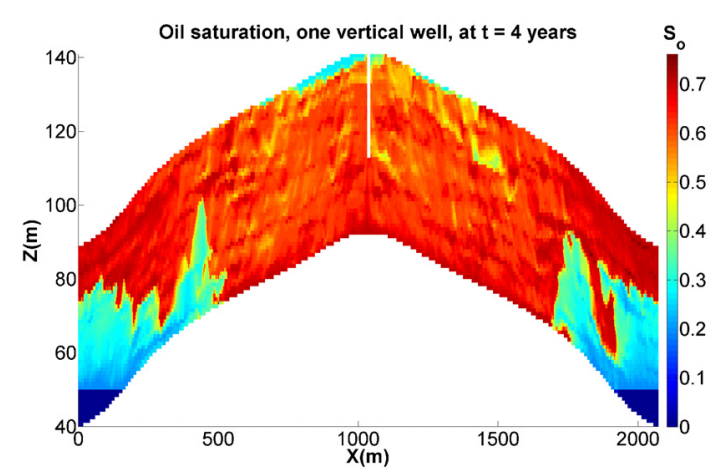

Figure 7 Oil saturation for initial vertical well configuration after 4 years of production.

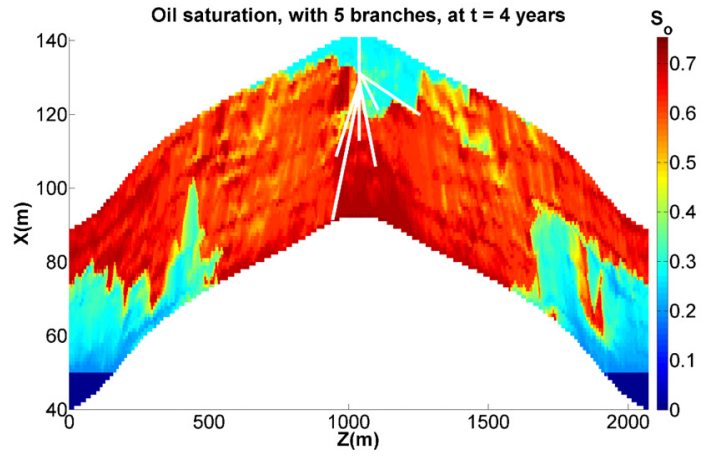

Figure 8 Oil saturation for optimized well trajectory with 5 branches after 4 years of production.

cubic meters $(324 \mathrm{Mbbl})$. This means an improvement of $13.7 \%$ with respect to the initial configuration with one vertical well.

Despite the addition of branches, we can observe on Figure 8, that there is still oil not produced in regions too far to be reached by a branch. Thus, we should revise the model and add the possibility to add vertical production wells when an area is too far from existing wells.

On this very simple case, we analyze the fluid flow simulator outputs corresponding to the end of the production time, but it may be interesting to compute scores at different periods when the oil production starts to decline.

\section{Conclusions}

In this paper we have proposed a two-step optimization methodology, to better determine both locations and trajectories of wells (branches) to maximize oil production. Promising results were obtained for a relatively simple example where the number and trajectory of the branches of a production well were optimized.

In future work, we will test the methodology on more realistic cases, the Brugge Benchmark (Peters et al. (2010)), based on a three-dimensional model with 10 injectors and 20 producers. For this more complex latter problem, heuristics methods, such as Variable Neighborhood Search (VNS, Liberti et al. (2011)) may perform better than Branch-and-Bound in terms of computational speed.

From our first results, the branching problem model should be review to have the possibility to add vertical production wells if remaining oil cannot be reached by a branch associated with an existing well in the neighborhood. This would increase the size of the branching problem, thus we have to better manage the number and relative location of branches for each well. The model could also be improved by accounting for oil along a branch for the computation of produced oil associated with branches.

Another enhancement of the approach presented in this paper could be related to the connectivity measure considered (a distance criterion in this work). Alternative measures could be determined using the permeability field distribution, or a simplified/fast-to-evaluate fluid flow simulator.

\section{Acknowledgments}

We would like to acknowledge David Echeverría Ciaurri for his precious advices. We also thank Eric Flauraud, Guillaume Enchéry, and Steven Thomas for their help in reservoir simulation. 


\section{References}

AbdulKarim, A., Al-Dhubaib, T., Elrafie, E. and Alamoudi, M. [2010] Overview of saudi aramco's intelligent field program. SPE Intelligent Energy Conference and Exhibition, Jaarbeurs, Utrecht, The Netherlands, March 23-25, SPE.

Audet, C. and Dennis, J.J.E. [2001] Pattern search algorithms for mixed variable programming. Siam J. Optim., 11(3), 573-594.

Bonami, P., Biegler, L.T., Conn, A.R., Cornuéjols, G., Grossmann, I.E., Laird, C.D., Lee, J., Lodi, A., Margot, F., Sawaya, N. and Wächter, A. [2008] An algorithmic framework for convex mixed integer nonlinear programs. Discrete Optimization, 5(2), 186-204.

Bouzarkouna, Z., Ding, D.Y. and Auger, A. [2010] Using evolution strategy with meta-models for well placement optimization. CoRR, abs/1011.5481.

Chiles, J.P. [1984] Simulation of a nickel deposit: problems encountered and practical solutions.

Dakin, R.J. [1965] A tree search algorithm for mixed-integer programming problems. Computer Journal, (8).

Echeverría Ciaurri, D., Isebor, O. and Durlofsky, L. [2011] Application of derivative-free methodologies to generally constrained oil production optimisation problems. International Journal of Mathematical Modelling and Numerical Optimisation, 2(2), 134-161.

Fourer, R., Gay, D.M. and Kernighan, B.W. [2003] AMPL : a modeling language for mathematical programming / Robert Fourer, David M. Gay, Brian W. Kernighan. Pacific Grove, CA : Thomson/Brooks/Cole, 2nd edn., ISBN 0534388094.

Gramacy, R. and Le Digabel, S. [2011] The mesh adaptive direct search algorithm with treed gaussian process surrogates. Tech. rep., Les Cahiers du GERAD G-2011-37.

Gupta, O.K. and Ravindran, V. [1985] Branch and bound experiments in convex nonlinear programming. Management Science, 31(12), 1533-1546.

Isebor, O.J., Echeverría Ciaurri, D. and Durlofsky, L.J. [2013] Generalized field development optimization using derivative-free procedures. Society of Petroleum Engineers Journal.

Le Digabel, S. [2011] Algorithm 909: Nomad: Nonlinear optimization with the mads algorithm. ACM Trans. Math. Softw., 37(4), 44:1-44:15, ISSN 0098-3500, doi:10.1145/1916461.1916468.

Le Ravalec, M., Tillier, E., Da Veiga, S., Enchery, G. and Gervais, V. [2012] Advanced integrated workflows for incorporating both production and 4d seismic-related data into reservoir models. Oil Gas Sci. Technol. - Rev. IFP Energies nouvelles, 67(2), 207-220, doi:10.2516/ogst/2011159.

Le Ravalec, M., Noetinger, B. and Hu, L.Y. [2000] The fft moving average (fft-ma) generator: An efficient numerical method for generating and conditioning gaussian simulations. Mathematical Geology, 32(6), 701723

Liberti, L., Mladenović, N. and Nannicini, G. [2011] A recipe for finding good solutions to minlps. Mathematical Programming Computation, 3(4), 349-390, ISSN 1867-2949, doi:10.1007/s12532-011-0031-y.

Matheron, G., Beucher, H., de Fouquet, C. and Galli, A. [1987] Conditional simulation of the geometry of fluviodeltaic reservoirs. Ann. Techn. Conf. and Exhib., Dallas, TX, SPE 16753.

Oliver, D., Reynolds, A. and Liu, N. [2008] Inverse theory for petroleum reservoir characterization and historymatching. Cambridge University Press, New York, USA.

Onwunalu, J.E. and Durlofsky, L.J. [2010] Application of a particle swarm optimization algorithm for determining optimum well location and type. Computational Geosciences, 14, 183-198.

Peters, L., Arts, R., Brouwer, G., Geel, C., Cullick, S., Lorentzen, R., Chen, Y., Dunlop, N., Vossepoel, F., Xu, R., Sarma, P., Alhuthali, A. and Reynolds, A. [2010] Results of the brugge benchmark study for flooding optimization and history matching. SPE Reservoir Evaluation \& Engineering, 13, 391-405, doi:10.2118/119094-PA.

PumaFlow [2012] PumaFlow Reservoir Simulator Reference Manual. BeicipFranlab.

Schulze-Riegert, R. and Ghedan, S. [2007] Modern techniques for history-matching. 9th International Forum on Reservoir Simulation, Abu Dhabi, UEA, December 9-13.

van den Berg, F., Perrons, R., Moore, I. and Schut, G. [2010] Business value from intelligent fields. SPE Intelligent Energy Conference and Exhibition, Jaarbeurs, Utrecht, The Netherlands, March 23-25, SPE. 